\title{
A Novel Genetic Convolutional Neuro Multi-Fuzzy Techniques for Newborn Face Recognition
}

\author{
T.Arul Raj ${ }^{1}$, Dr.R.Balasubramanian ${ }^{2}$ \\ ${ }^{1}$ Research Scholar, ${ }^{2}$ Professor \\ Department of Computer Science and Engineering, Manonmaniam Sundaranar University, Abishekapatti, \\ Tirunelveli. \\ Corresponding Author Mail id: arulraj121@gmail.com
}

Article History: Received: 11 January 2021; Accepted: 27 February 2021; Published online: 5 April 2021

\begin{abstract}
Advances in technology have made life simpler in today's society by supplying us with a variety of emerging demands lacking By assessing the progressive stability of biometric recognition accuracy for newborns, biometric recognition can be used to recognize missing newborns and prevent them from being switched in higherlevel hospitals.. Recognizing and authenticating newborns is a major problem in many hospitals. The face recognition system does an outstanding job of identifying and authenticating the newborn. To answer these concerns, create a face recognition device for newborns. The proposed approach improves picture consistency on a newborn's face. Our objectives are to propose a genetic, convolutional neural network, and fuzzy logic-based automated framework for newborn face recognition. As a paradigm GCNMF is suggested for real-world newborn face recognition. Convolutional, pooling, and fully-connected layers, as well as a Neuro Fuzzy layer, form the Inherited Convolutional Neuro Multi-Fuzzy. The model employs hereditary, convolutional neural networks, and fuzzy logic to deal with ambiguity and imprecision in the input configuration representation. The efficacy and outcomes of the recommended method are then analyzed using newborn face datasets and the Genetic Convolutional Neuro Multi-Fuzzy (GCNMF) Approach.
\end{abstract}

Keywords: Newborn face recognition, Convolutional Neural Network (CNN), Neuro Fuzzy (NF), Genetic Convolutional Neuro Multi-Fuzzy (GCNMF).

\section{Introduction}

Understanding biometrics and biometric security systems on a basic level will help you understand the definition of network security more easily. Biometrics [21] refers to the basic physical/logical features or attributes of the human body. These attributes and qualities help to determine who you are. Face, retinal, iris, fingerprint, palm print, and DNA are examples of unique biometric data that will be used to determine a person's identity (ID). Biometric devices can capture and store this information in order to verify personal identity. Biometric data systems are combined with biometric recognition and identification technologies to build biometric security systems. The biometric security system is a lock and capture system that limits access to personal data. In order to gain access to the biometric security system, individuals must have their unique characteristics or attributes compared to a database in the system. The locking method will grant the user access to the data if a match is found. The locking and capturing system will be turned on, and information about users who have accessed the data will be kept on file. The partnership between biometrics and biometric security is referred to as the lock and key scheme. The lock is the biometric security system, and the key is biometrics [20].

One of the simplest features that be able to be used in biometric security systems towards recognize a consumer is the human face. Face recognition technology is identical common and commonly used because it does not require any physical interaction between the user and the computer. For authentication, cameras check the user's face and compare it to a database. It's also easy to set up and doesn't necessitate any high-priced hardware. Facial recognition is commonly used in security systems such as physical access control and device user accounts. It is, however, not as distinctive as retinal, iris, or DNA. As a consequence, it's typically used in combination with other device characteristics. Face recognition technology, on the other hand, is most negatively affected by time because the user's age changes over time [10].

Wireless technology is used to monitor and track newborns in the Newborn Safety system. A wireless receiver is attached to the newborn's ankle to monitor its schedules inside the hospital, ensuring that the baby is still being cared for by the correct person and with the correct mother. Newborn robberies have become more frequent in recent years, leading hospitals to invest in sophisticated automation detection and safety systems that would not only secure newborn babies in hospitals, but also ensure that they were paired with the correct mother. Adults were studied to see how accurate Fingerprints, ears, and iris were three of the most frequently used biometric features. However, the long-term accuracy of biometric identification in children aged 0 to 4 years has not been extensively investigated. Given that young children are often disobedient and do not comply, understand or obey orders, fingerprints are the most sustainable biometric modalities for identifying offspring, in our view. This is due to the fact that capturing fingerprints of young children is easier than capturing other biometric features such as iris, which requires a kid towards look openly at the camera to begin iris capture. Our study into the 
persistence of fingerprint recognition in children aged 0-4 years is detailed in this article. Based on primary results acquired from data together during the first part of our study, fingerprints appear to be a promising method for recognizing children aged 0 to 4 .

While biometric features-based structures function glowing for confirming and recognizing adults, identifying and authenticating newborns, infants, and toddlers with high accuracy using biometric structures remains a difficult issue. Due to a deficiency of security processes in hospitals and intensive care units, accurate identification of newborns, babies, and toddlers is becoming increasingly necessary [6]. Infant switching, hospital abductions, erroneous baby transfers, and even inappropriate drug deliveries in hospitals have all been identified in various instances. He [13] discovered that 50 percent of newborn babies in the United States are misidentified (out of 34). Per year, between 1,00,000 and 5,00,000 newborn babies are mistakenly traded in the United States. There have been several reports of baby abduction and illegal adoption in China [14]. Every year in the United States, about 800,000 children go missing. Owing to a deficiency of accurate identification and authentication methods [17], it is difficult to locate or recognize such a missing child. According to UNICEF [11], 6.6 million children die each year as a result of not receiving enough vaccinations. To resolve such concerns for newborns, an effective system must be established that can classify the newborn and keep track of the vaccines that have been administered.

The following is how this paper organizes the design of an effective newborn face recognition system: Section II delves into the history and current research on face, iris, footprint, and fingerprint recognition systems for newborns, babies, and toddlers, while Section III delves into the face recognition database. The fuzzy system for newborn face recognition is introduced in section IV. The proposed system's performance and efficiency results are presented in section $\mathrm{V}$, and our research work is concluded in section VI with a reference.

\section{Related Work}

Biometric systems determine identification by analysing an individual's physical and behavioural characteristics, such as fingerprints, face, hand geometry, iris, gait, and voice. Travel and transportation, financial institutions, health care, law enforcement agencies, and different government sectors can all use the services of a biometric system [16]. With the development of ICT-enabled technology, a high-reliability validation and right to use mechanism is required. In many ICT-enabled technologies, biometric features such as fingerprints, faces, DNA sequences, palms, and iris have been commonly used for identifying and authenticating adults. Biometric images such as fingerprints, faces, and iris are collected from people who use the sensor during enrollment and preprocessed to extract specific features using various feature generation/extraction techniques. After that, certain characteristics are filtered in order to create and store individual templates.

For newborn personal authentication, a fundamental online footprint recognition approach is proposed. They proposed a texture feature extraction-based recognition system after developing preprocessing techniques for together standard and special context conditions. The device outperforms offline footprint recognition systems, which had only a $1 \%$ to $20 \%$ identification accuracy. As a result, biometric data from the feet may be a very useful method for newborn identification. The use of a multimodal method to identify newborns improves the identification process [8]. The data generated by each unimodal modality is combined to produce a concluding evaluation in multimodal biometric data fusion of various biometric modalities. Multimodal fusion has been shown to enhance the recognition system's robustness, enabling it to make a recognition decision even though one or more biometric decisions are unsuccessful. It is more successful to use a fusion scheme early in the recognition method. The choice of normalization technique affects the efficiency of sum rule-based fusion. Infant abductions, illegal adoptions, child swapping in hospitals after birth, and baby girl murders are all on the rise, posing a threat to society. Efficient, timely, and robust steps are needed to prevent such incidents [7]. Biometrics-based identity authentication, which uses online image collection, electronic processing, and storage to solve these disadvantages, is proposed as a solution. As a consequence, rather than using traditional ink and paper methods, an online process is used. Biometric face recognition systems can gather information from users' faces and store it in a database for later use. It will assess the overall form, shape, and proportion of features on the user's face, such as the gap between the user's eyes, nose, mouths, ears, and jaw, as well as the scale of the user's eyes, mouth, and other expressions. One of the variables that can alter during a user's facial recognition process is his or her facial expression. Smiles, tears, and lines on the face are only a few examples [3].

Because of rising security threats and commercial fraud, as well as the advancement of personalization and accessibility, the importance of biometrics as a reliable tool for individual recognition has been established beyond doubt. They [2] attempted to summarize the current state of biometric recognition and identified key concerns that must be addressed. Biometrics has come a long way in the last 50 years. On the one hand, tremendous progress has been made in the design of large-scale biometric systems that can quickly search biometric databases for a matching identity. However, with the introduction of smartphones and other consumer devices, there has been renewed interest in developing biometric solutions for devices with limited resources (e.g., the Touch ID fingerprint system in iPhones). Modern biometric systems are increasingly dealing with low-quality data. Such as those used in forensics. Improvements in computing power have assisted signal processing, 
computer vision, pattern recognition, and machine learning. Despite continuing challenges, the biometrics community can be proud of its accomplishments over the last 50 years.

Recent studies have suggested algorithms for newborn face recognition, primarily oriented toward the use of non-intrusive face picture capture to enroll newborn individualities at birth and validate them before they leave the hospital. He has taken face descriptions of newborns (0-3 weeks old) [31], and he is especially interested in the complex processes that are used to classify infants using a novel intelligent technique. SVM divides groups by fuzzier boundaries than neural networks, which use only single lines to distinguish them. Furthermore, a neural network with 40 classes is unable to find a generalized mapping feature capable of correctly classifying all of the data. We may infer from the results that SVM outperforms the neural network by over 10\%. Owing to their gross head reflexes, posture, and speech changes, newborns make it difficult to capture high-quality face pictures. 9th Formal jargon Records rank-1 proof of identity accuracy of 78.5 percent and authentication accuracy of 63.4 percent in a gallery of 86 subjects, with a 0.1 percent false accept rate (FAR) on four photographs per newborn (all face images were cropped using manually annotated eye and mouth locations). According to the results, the proposed face recognition technology outperforms current algorithms and commercial systems on a newborn face database obtained from multiple hospitals in challenging environments. Although face recognition may not be capable of meeting the accuracy requirements for managed applications such as healthcare, it is still important to evaluate efficiency and recognize issues, and it may be an useful semi-automated investigative tool for certain law management solicitations (e.g. finding lost children). Given offenders' high levels of motivation, this problem is crucial, and it is still an open research question.

The efficiency and performance of the proposed system was assessed and analysed using a child biometrically compatible (CMBD) database [15] as well as a tried-and-tested NITG fingerprint database. The main objective of this research is to reduce the number of missing or changed newborns: How to find children missing? Every year in the United States, over 800,000 children are missing, and every 40 seconds, one children are missing. We want first of all to see how long it takes for children aged 0 to 4 to recognize the fingerprints. Based on preliminary data obtained during the first phase of our research, it appears promising to use fingerprints for identifying 0-4-year-olds. The use of fingerprints for child and child identification appears promising on the basis of our preliminary findings. In September 2015 (Phase II), December 2015 (Phase III) and March 2016 (Phase IV), we plan to collect longitudinal data from the same subjects three times more over a one-year period to examine thoroughly the persistence of fingerprint recognition in children between 0 and 4 years of age [13]. It is necessary to control and connect their traits with their mothers to prevent newborn switches. To avoid newborn switching. The only disadvantage is that many hospitals use RFID bracelets for the reason is the easy loss and exchange of them. As a result, biometrics have been advocated for use in monitoring newborns. To determine which biometric trait can be used to reliably identify newborns, ongoing research efforts are needed.

An overview of the fingerprint biometric system is given, as well as information on the system's current security aspects. Among the security challenges discussed are multiple device attacks, relevant threat models, biometric cryptosystems, present issues, risks, prospects, and exposed problems in modern fingerprint biometric systems [23]. We primarily motivated on providing biometric systems-related topics that are sufficient to provide the reader with a framework and allow them to further investigate and contribute to the field of biometric security. Fingerprint-based recognition systems are the most widely researched and implemented biometric traits (e.g., face, iris, and voice). The pattern of valleys and ridges that make up a fingerprint is determined after birth, and even identical twins may have different fingerprint patterns [19]. The accuracy of fingerprint-based recognition systems has been stated to be very high [24], with public acceptance of fingerprint acquisition being moderate [18]. As a result, fingerprint biometric devices have a sizable market share and are used in a variety of applications. While fingerprint recognition has a promising future, it is plagued by problems such as inadequate accurateness and safety concerns.

When used popular unimodal authentication, the equal error rate weighted (EERW) form had weights comparative to the EERs for each modality. Weights were determined using the distribution of the modalities' corresponding score in the distribution weighted (DW) process. For the purpose of determining the optimal weights, we used a Fuzzy Adaptive Genetic Algorithm (FAGA) [12]. As compared to existing weight estimation methods, the use of optimized weights has resulted in better authentication efficiency. The weight used for score fusion for these two modalities was optimized using a fuzzy adaptive genetic algorithm in this paper. It is possible to note this.

\section{Dataset Description}

The newborn dataset is made up of photographs of 181 newborn faces (Fig.1), which correspond to 16 subjects [5]. It was gathered from various publicly available sources. Each subject is represented by a minimum of four photographs. Male and female subjects, ranging in age from a few days to six months, are represented in the dataset.

Illumination, picture consistency, voice, pose, ageing, and disguise are all significant covariates in newborn face recognition. The tasks of ageing and disguise do not exist in the case of a newborn. Expression is 
the most distinguishing covariate between adult and infant faces. The apparent geometrical shape and location of a facial feature is influenced by facial expression; however, the effect on recognition may be greater for geometrybased methods than for holistic methods.

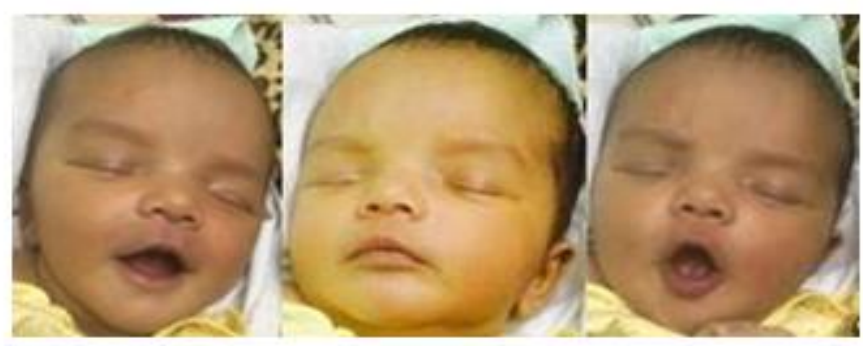

Fig.1. Sample of Newborn images

Furthermore, 3-5 face images were collected for each individual child (181 face images from 16 subjects) to see if the face could be used as another cue to improve global recognition performance. Due to throughput constraints, face image capture was also relatively unrestricted; lighting, stance, and expression remained not controlled during the face apprehension process.

\section{PROPOSED METHODOLOGY}

The proposed Genetic Convolutional Neuro Multi-Fuzzy (GCNMF) (Fig.2), a convolutional neural network with fuzzy inputs for newborn baby face recognition using LDA-DCP [4] features derived from newborn faces, is described in this paper. The genetic algorithm [3] is used to choose the optimized features, and the optimized futures are added to both the CNN and the Neuro fuzzy algorithms separately. Then, to boost the recognition result, a multi-fuzzy package containing CNN and Neuro fuzzy output is fed into the fuzzy system. The parts that follow provide additional background.

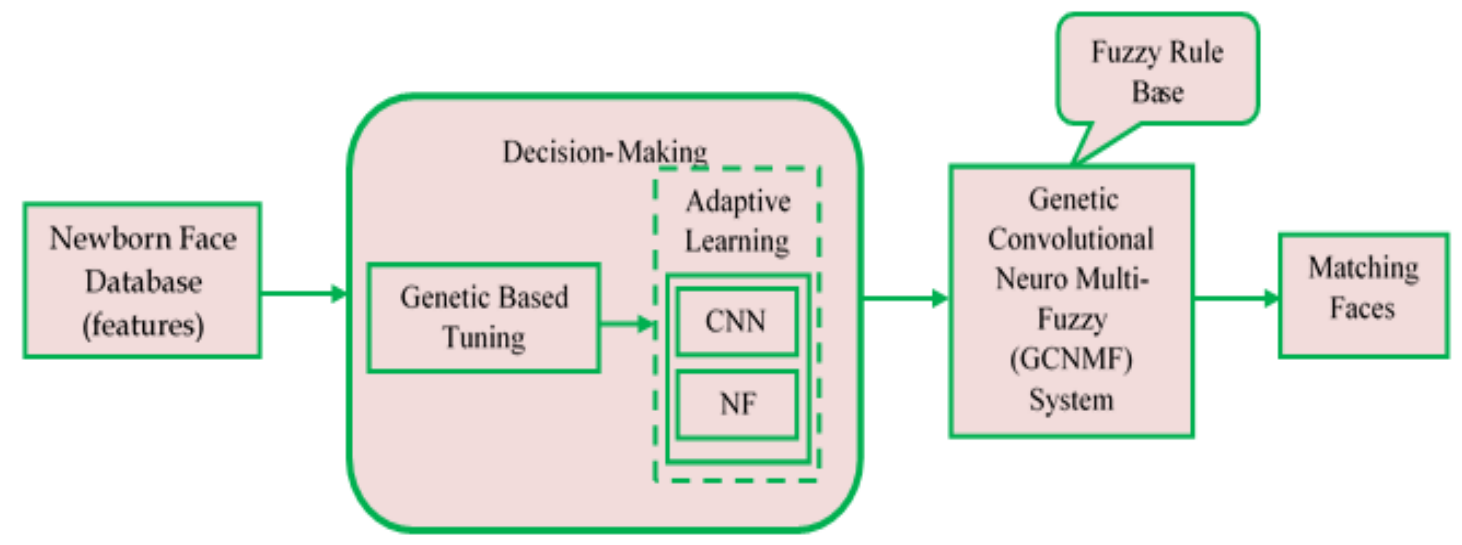

Fig.2. General Structure of proposed recognition control system based on Genetic Convolutional Neuro Multi-Fuzzy

During an operation, four distance measurements are measured based on the newborn's tracking data. The basic elements and for each action, the range of variations of these distance measures is used to construct fugitive membership functions that highlight each action's discriminatory position. In these fuzzy membership functions the temporal variation in the membership values is characteristic of the recognition of man's behaviour. A neural network, accomplished of identifying local patterns in input data, is used to identify human actions.

4.1 Convolutional Neural Network

Convolutional neural networks are neural networks that use convolution instead of general matrix multiplication in at least one layer. A convolutional neural network is composed of an input and output layer (Fig. 3 ) as well as multiple hidden layers [27]. The activation function and final convolution are normally accompanied by additional convolutional layers, such as the normalization layer, max pooling layer, and fully connected layer, which are referred to as hidden layers because their inputs and outputs are covered by the activation function and final convolution. Back propagation is commonly used in the final convolution to ensure that the end product is correctly weighed. 


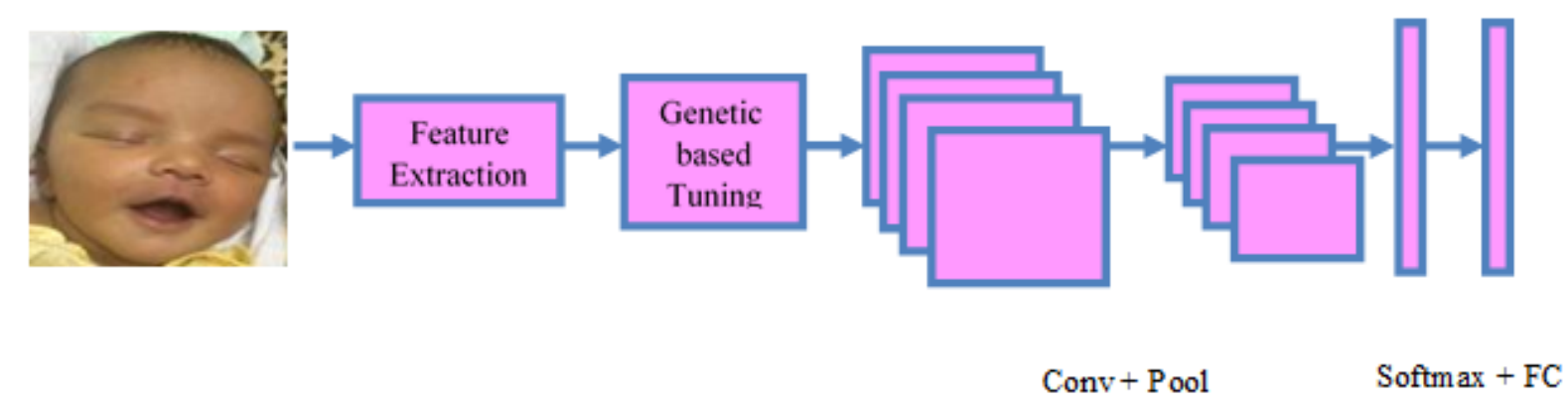

Fig.3. General Architecture of CNN for Newborn Face Recognition

\subsection{Neuro Fuzzy Classification}

Things that are not obvious or ambiguous are referred to as fuzzy. In the real world, we often come across situations in which we are unable to decide if a state is true or false; their fuzzy logic offers a great deal of reasoning versatility. This allows us to take into account any situation's inaccuracies and uncertainties. It's a tool for integrating human-like thought into a computer control system [29]. It isn't designed to be precise, but it is designed to be appropriate. It may mimic human deductive reasoning, or the mechanism by which people draw conclusions based on what they know. With the aid of fuzzy logic, any uncertainties can be easily addressed. The absolute truth value in a boolean method is 1.0 , while the absolute false value is 0.0 . However, there is an intermediary assessment in fuzzy logic that is both true and false. There are four parts to its structure:

- RULE BASE: This section includes the experts' collection of rules and IF-THEN conditions for governing the decision-making mechanism using linguistic data. Several effective methods for the design and tuning of fuzzy controllers have recently emerged from recent advances in fuzzy theory. The bulk of these advancements have resulted in a decrease in the number of vague laws.

- FUZZIFICATION: This is a technique for converting crisp numbers into fuzzy sets. Crisp inputs, such as temperature, pressure, and rpms, are the exact values calculated by devices and transferred to the control system method for handling.

- INFERENCE ENGINE: It computes the unit of match between the recent fuzzy input and every rule, and then determines the rules should be executed based on the input field. The control actions are then generated by integrating the fired rules.

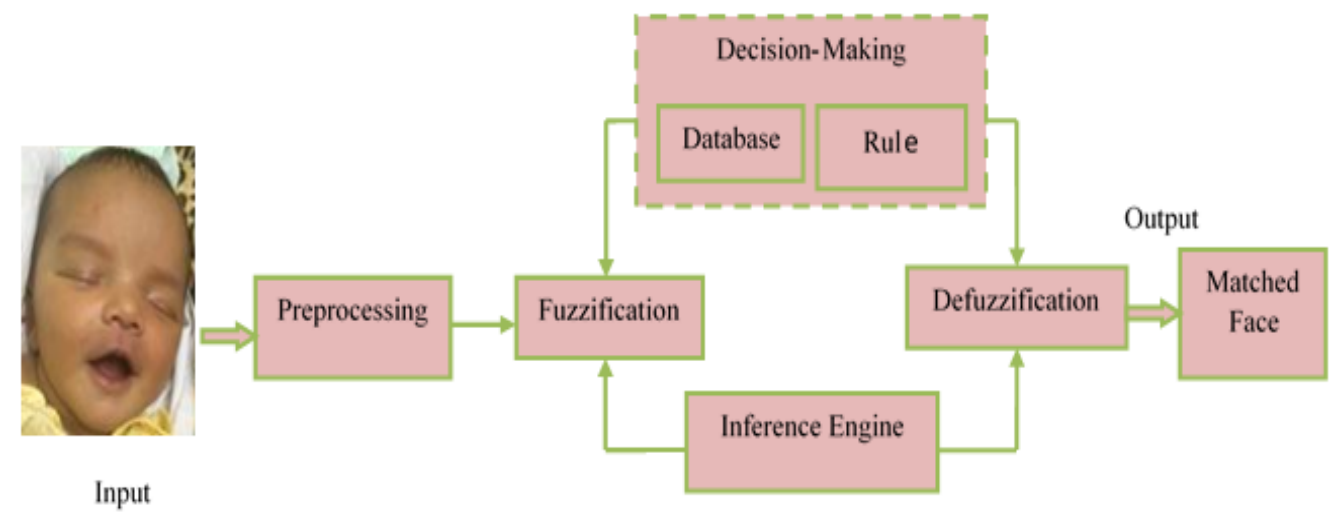

Fig.4. General Architecture of Neuro Fuzzy for Newborn Face Recognition

- DEFUZZIFICATION: It's used to transform the fuzzy sets created by the inference engine into a crisp value. There are many defuzzification approaches to choose from, and the best one is used in conjunction with a particular expert framework to minimise error. 
After collecting photographs from the same party, the fuzzy crisp values of their face pixels with relevant parts, such as the eyes, nose, lips, and so on, are taken into account [28]. On the basis of these crisp values (Fig.4), images in the group and the input image are actual measurements. The sum of matching for each part is used to determine a ranking. A higher score is awarded to the better player. The use of a scoring system means that each component is given equal weight. Each variable has its own set of weights. Nose, lips, eyes, and brows all have a certain amount of weight. Even all the same the region with different face parts in those two images differs, a newborn with two different expressions in two images would be paired as one newborn in this way.

4.3 Proposed Genetic Convolutional Neuro Multi-Fuzzy

The recommended system is a smart scheme that incorporates intelligent technology such as genetic algorithms, convolutional neural networks, and fuzzy logic (Fig.5). These systems have a broad range of capabilities due to the integration of various techniques into a single computational model. In an unpredictable and imprecise world, these systems are able to reason and learn. These systems can have human-like capabilities such as domain intelligence, noise adaptation, and so on. A Neuro Genetic hybrid system combines neural networks, which can learn from examples, recognize objects, and shape relationships between them, with genetic algorithms, which can perform important search and optimization techniques. To boost the efficiency of neural networks, genetic algorithms can be used. and to evaluate the input relation weights. These algorithms can be used to choose topologies and train networks as well. A Fuzzy Genetic Hybrid System is being developed to improve and model Genetic algorithms using fuzzy logic based techniques. The genetic algorithm has proven to be a reliable and efficient method for generating fuzzy rule bases, membership functions, and other tasks.

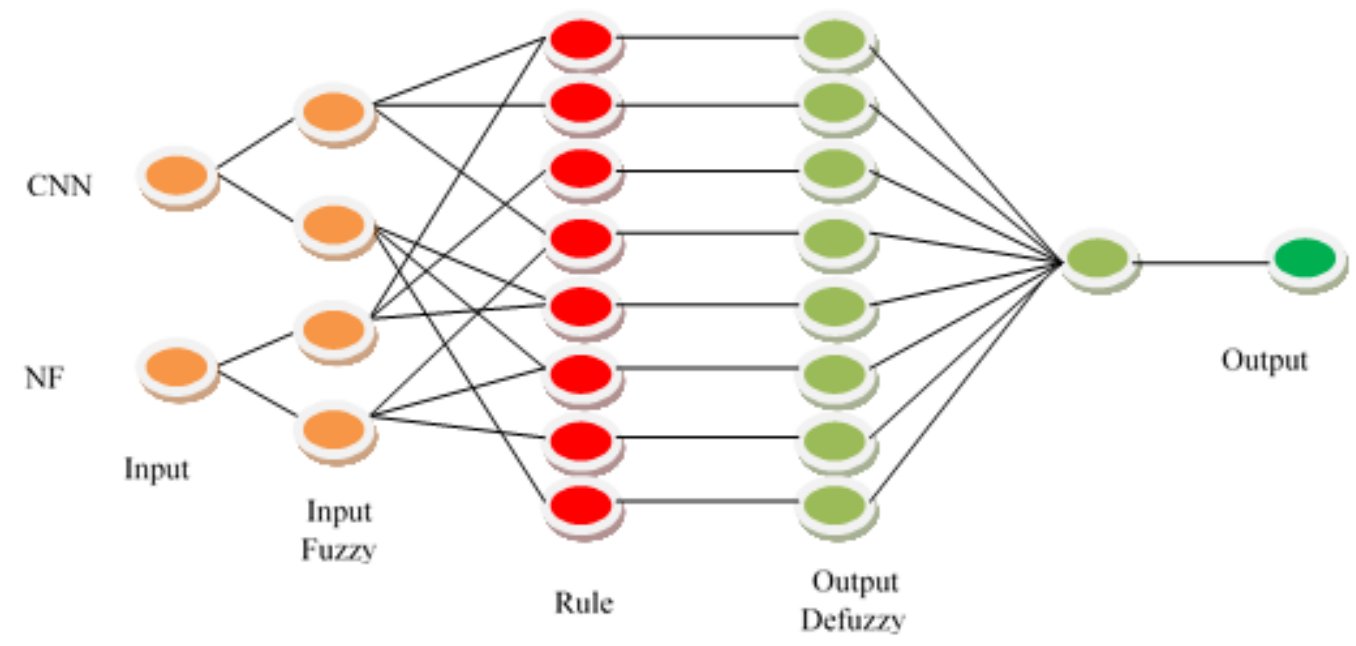

Fig.5. Proposed Architecture for Genetic Convolutional Neuro Multi-Fuzzy

Various combinations of genetics, neural networks, and fuzzy logic exist. Traditional neural networks may have fuzziness inserted into various sections. One of the most common ways to integrate fuzziness into network structure is to apply fuzziness to the values of learning instances, "blur" input data, and extract output information using fuzzy sets theory [22]. The paper proposes a typical for real-world object and scene image classification called Genetic Convolutional Neuro Multi-Fuzzy (GCNMF). The proposed Genetic Convolutional Neuro Multi-Fuzzy (GCNMF) model has five layers: genetic, convolutional, pooling, fuzzy, and completely linked.

Algorithm for the GCNMF

- Begin by creating a first-generation population of solutions.

- Class labels for the input features to the fuzzy system are generated by the CNN and NF classifiers.

- Build a rule based fuzzy system and use the fuzzy rule to measure output indexes.

- Neurons representing fuzzy sets are fed into the fuzzy rule layer. using the fuzzy

- Each defuzzification neuron represents a single neuro-fuzzy system output and integrates all inputs operation UNION.

- Finally, the matched faces are determined using the genetic convolutional fuzzy system.

\section{Experimental Result and Analysis}

Even though the corresponding normal $\mathrm{CNN}$ does not improve the quality of classification problem solution (accuracy), incorporating CNN and NF into the Fuzzy Layer with the mamdani form (Fig. 6) helps to improve the standard of classification problem solution (accuracy). As a result, the proposed GCNMF helps to 
increase their profile. To assess the efficacy of newborn baby face recognition, we manually picked one face picture for each subject for the gallery. The remaining 181 newborn baby face photographs from the 16 subjects were analysed. Despite the fact that these newborn face matching results are less than satisfactory, face images are still useful operationally, particularly given that they were acquired on the same day (in fact, the same minute).

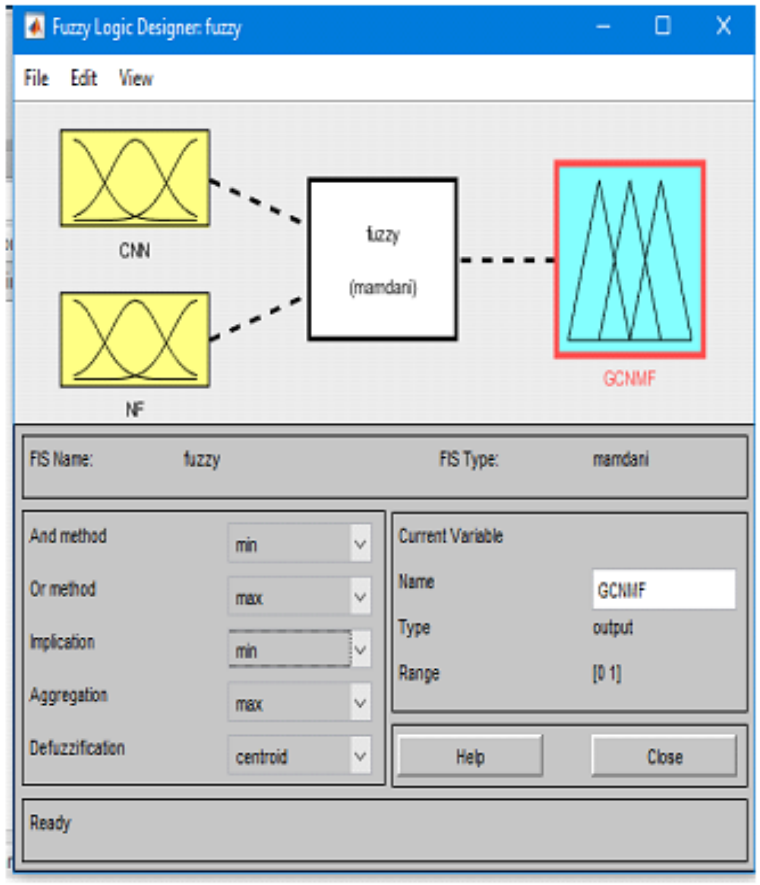

(a)

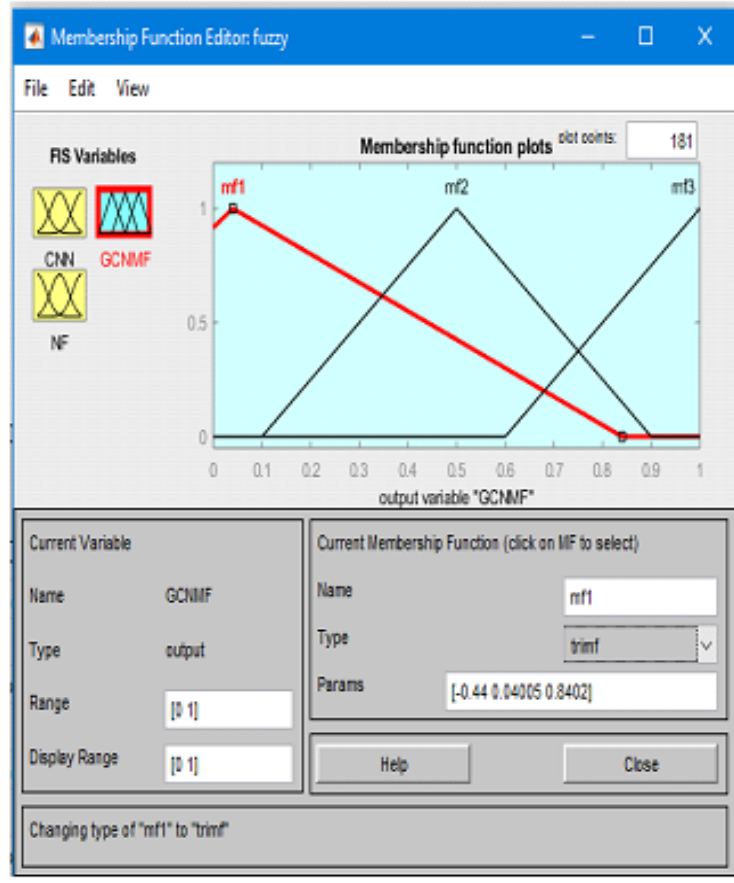

(b)

Fig.6 Implementation of Proposed GCNMF: (a) loading input \& (b) Assiging Member Fuctions.

Table.1. Comparison of Accuracy, Sensitivity, Specificity, PPV, NPV using GCNMF

\begin{tabular}{|c|c|c|c|}
\hline $\begin{array}{c}\text { Performance } \\
\text { M etrics }\end{array}$ & CNN & NF & $\begin{array}{c}\text { Proposed } \\
\text { GCNMF }\end{array}$ \\
\hline Accuracy & 98.10 & 97.99 & 99.05 \\
\hline Sensitivity & 100 & 100 & 100 \\
\hline Specificity & 93.45 & 89.58 & 95.85 \\
\hline PPV & 80.23 & 69.25 & 90.32 \\
\hline NPV & 100 & 100 & 100 \\
\hline
\end{tabular}




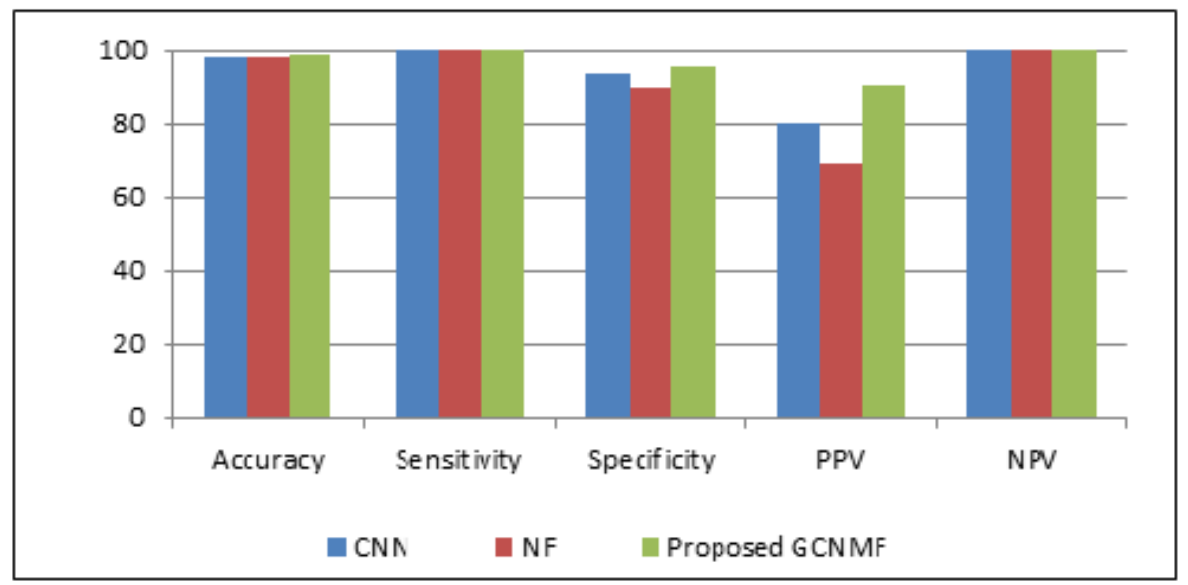

Fig.7. Comparison aralysis of performence metrics (Accuracy, Sensitivity, Specificity, PPV, NPV)

Table 1 shows that the proposed method, GCNMF with optimized function subset, improves classification efficiency and achieves the best recognition accuracy of 99.05 percent. Other performance metrics such as accuracy, sensitivity, specificity, Positive Predictive Value (PPV), and Negative Predictive Value (NPV). For a better example, the GCNMF efficiency metrics are shown in (Fig.7).

Table.2. Comparison of F-Measure, ROC, G-Mean, MCC using GCNMF

\begin{tabular}{|c|c|c|c|}
\hline $\begin{array}{c}\text { Performance } \\
\text { M etrics }\end{array}$ & CNN & NF & $\begin{array}{c}\text { Proposed } \\
\text { GCNMF }\end{array}$ \\
\hline F-Measure & 94.23 & 93 & 95.47 \\
\hline ROC & 95.63 & 94.57 & 96.08 \\
\hline G-Mean & 94.58 & 93.74 & 96.74 \\
\hline MCC & 81.65 & 80.03 & 90.98 \\
\hline
\end{tabular}

Fig.8. Comparison analysis of performance metrics (F-Measure, ROC, G-Mean, MCC)

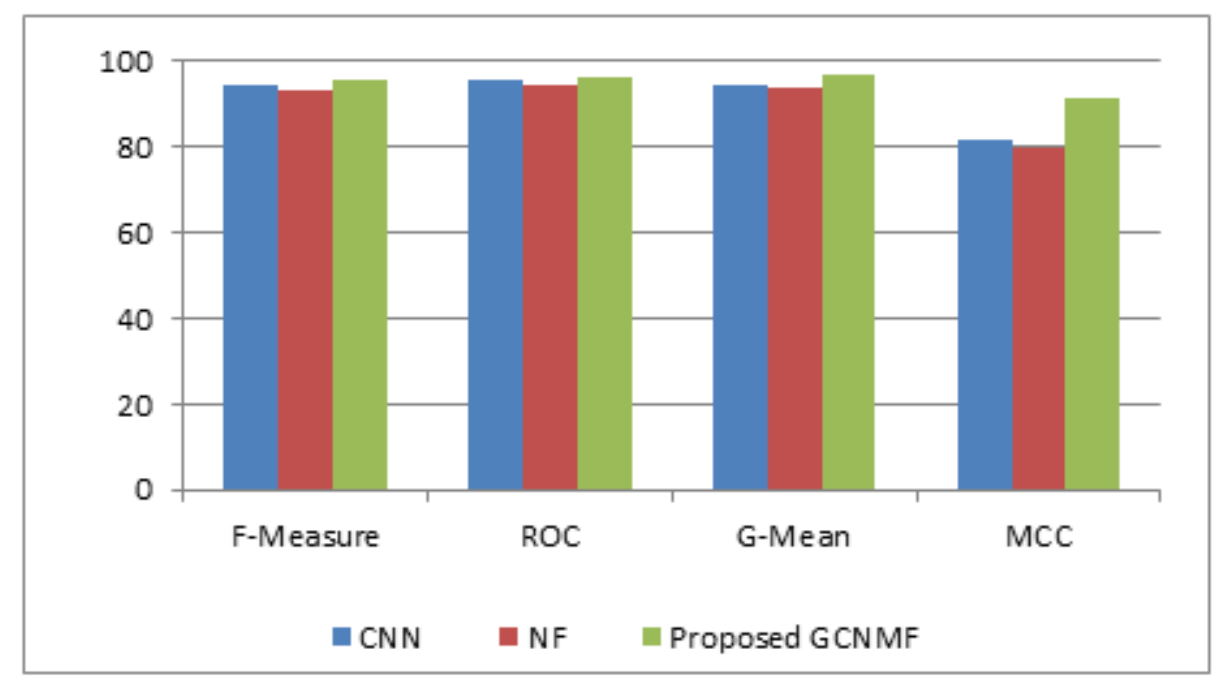


The method proposed measures the worth of performance metrics. Other performance metrics such as F-Measure, Receiver Operating Characteristic Curve (ROC), G-Mean, and Matthews Correlation Coefficient (MCC) are also considered, and GCNMF with optimised function subgroup is used to achieve the best recognition rate (Table 2). Finally, the proposed GCNMF (Fig.8) is the best classifier fuzzy system and provides the best results when using the newborn face database's optimised function subset.

\section{Conclusion}

There is an increasing need for developing biometric capabilities to identify newborn babies. Distinctiveness and durability belongings of a biometric trait must met for the people awareness in order for biometric recognition to be effective. Although these two biometric principles has studied form the four major biometric traits (face, footprint, fingerprints, and iris) of the newborn baby has no systematic longitudinal research has been undertaken to examine the persistence of biometric identification for newborn babies. This paper proposes a new expert method for identifying lost and exchanging newborn babies established on a fuzzy system with CNN and NF high potential points. The proposed framework used fuzzy logic rules to detect missing and swapping newborns, transforming it into an expert control system. Our proposed system now has a 99.05 percent accuracy rate for newborn face recognition. The missing strength rate is determined by this expert method, making identification more reliable and complete. As a result, this paper introduces the GCNMF paradigm for newborn baby face recognition. According to fuzzy sets theory, fuzzyness is integrated into the network structure. The proposed model is capable of dealing with insecurity and imprecision because it incorporates the strength of hereditary, convolutional neural networks, and fuzzy logic. The efficiency of GCNMF is evaluated then the findings indicate that the GCNMF can provide the enhanced accuracy in a smaller amount of training time.

\section{REFERENCES}

1. Anil K. Jain, et.al, "Biometrics for Child Vaccination and Welfare: Persistence of Fingerprint Recognition for Infants and Toddlers", MSU Technical Report, MSU-CSE-15-7, April, 15, 2015.

2. Anil K. jain, K. Nandakumar and A. Ross. "50 years of biometric research: Accomplishments, challenges, and opportunities." Pattern Recognit. Lett. 79, 80-105, 2016.

3. Arul Raj T. and Dr.R.Balasubramanian, "A Novel Hybrid Genetic Wolf Optimization for Newborn Baby Face Recognition”, Paideuma Journal, ISSN No: 0090-5674, Vol XIII Issue II 2020.

4. Arul Raj T. and Dr.R.Balasubramanian, "Fusing LDA- DCP Feature Sets for Newborn Baby Face Recognition using Feature Selection and Classification using Deep Convolutional Neural Network", Compliance Engineering Journal, ISSN: 0898-3577, Volume 10, Issue 11, 2019.

5. Arul Raj T., A.Gopi Kannan and Dr.R.Balasubramanian, "Empirical Analysis for Newborn baby Face Recognition with Ensemble Feature Extraction and Classifier", Journal of Information and Computational Science, ISSN: 1548-7741, Volume 9 Issue 10 - 2019.

6. Ashwini R. Patil ,Amol D. Rahulkar ,Chirag N. Modi, "Designing an Efficient Fingerprint Recognition System for Infants and Toddlers", 10th ICCCNT 2019 July 6-8, 2019.

7. Balameenakshi S., S. Sumathi, "Multi-Biometric System For Newborn Recognition", International Journal of Engineering Research \& Technology (IJERT), Vol. 2 Issue 5, ISSN: 2278-0181, May - 2013.

8. Balameenakshi S., S.Sumathi, "Biometric Recognition of Newborns: Identification using Footprints", Proceedings of 2013 IEEE (ICT 2013), 737-742, 2013.

9. Bharadwaj S., H. S. Bhatt, M. Vatsa, and R. Singh, "Domain specific learning for newborn face recognition," IEEE Trans. on Information Forensics and Security, vol. 11, no. 7, pp. 1630- 1641, July 2016.

10. "Biometrics new portal" UK http://www.biometricnewsportal.com/, 2011.

11. Committing to child survival: A promise renewed, Sep 2013

12. Gao L., F. Lu, Y. Ge, and D. Feng, "A fuzzy adaptive Genetic Algorithms for globaloptimization problems," in Control and Decision Conference (CCDC), Chinese, pp. 914 - 919, 2010.

13. Gray J.E., G. Suresh, R. Ursprung, W.H. Edwards, J. Nickerson, and P.H. Shinno, "Patient misidentification in the neonatal intensive care unit: Quantification of risk," Pediatrics, vol. 117, pp. 4647, 2006.

14. Illegal adoption.https ://www.unric.org /en/ humantrafficking/ 27450-illegal-adoption?

15. Image Analysis and Biometrics Lab @ IIIT Delhi. [Online] Available: http://www.iab-rubric.org /resources

16. Jain A. K., and A. Ross, Introduction to Biometrics, A. Handbook of Biometrics. Springer, pp. 1-22. 2008.

17. Jain A.K, Aroda A.K., Cao S.S., L. Best-Rowden, and A. Bhatnager, "Fingerprint recognition of young children," in IEEE Transactions on Information Forensics and Security, vol. 12, no. 7, pp. 15011514, July 2017. 
18. Jain, A.K., Ross, A., Prabhakar, S., "An introduction to biometric recognition", Volume: 14 Issue: 1, on pg: 4 - 20, Issue Date: Jan. 2004.

19. Jain, A.K.; Flynn, P.; Ross, A.A. Handbook of Biometrics; Springer: New York, NY, USA, 2007.

20. Jain, A.K.; Ross, A.; Pankanti, S., "Biometrics: a tool for information security" Volume: 1 Issue: 2, pg: 125 - 143, Issue Date: June 2006.

21. Jain, A.K.; Ross, A.; Prabhakar, S, "An introduction to biometric recognition”, IEEE Trans. Circuits Syst. Video Technol, 14, 4-20, 2004.

22. Kseniya P. Korshunova, “A Convolutional Fuzzy Neural Network for Image Classification”, 978-15386-7531-1/18/\$31.00, IEEE @2018.

23. Mahesh Joshi, Bodhisatwa Mazumda and Somnath Dey, "Security Vulnerabilities against Fingerprint Biometric System", Computer Science, Cryptography and Security, May 18, 2018.

24. Maio, D.; Maltoni, D.; Capelli, R.; Franco, A.; Ferrara, M.; Turroni, F. FVC-onGoing: On-Line Evaluation of Fingerprint Recognition Algorithms. 2013. (Accessed on 25 January 2019).

25. Naif Alajlan, Md Saiful Islam, Nassim Ammour, "Fusion of Fingerprint and Heartbeat Biometrics Using Fuzzy Adaptive Genetic Algorithm”, Conference: World Congress on Internet Security At London, UK (WorldCIS) 2013.

26. Preciozzi, Javier \& Garella, et.al, "Fingerprint Biometrics From Newborn to Adult: A Study From a National Identity Database System", IEEE Transactions on Biometrics, Behavior, and Identity Science. PP. 1-1, Jan 2020.

27. Sahar Siddiqui, Mayank Vatsa, and Richa Singh, "Face Recognition for Newborns, Toddlers, and PreSchool Children: A Deep Learning Approach" 24th ICPR (Conference), IEEE, 2018.

28. Senthil Kumar S., "Multimodal Biometric Technology Using Fuzzy Logic Decision and Fuzzy Inference System", Asian Journal of Computer Science and Technology ISSN: 2249-0701 Vol. 5 No. 2, pp.1-4, 2016.

29. Suchitra Basak1, Ruting Jia1, Chengwei Lei2, "Face Recognition using Fuzzy Logic", Proceeding of the IEEE International Conference on Information and Automation Wuyi Mountain, China, August 2018.

30. Tiwari S., A. Singh, and S. K. Singh, "Intelligent method for face recognition of infant", International Journal of Computer Applications, vol. 52, no. 4, pp. 36-50, 2012.

31. Wencheng Yang, Song Wang and et.al, "Security and Accuracy of Fingerprint-Based Biometrics: A Review”, MDPI, Symmetry, 11, 141, 2019. 